\title{
L'Engagement des femmes dans le monde associatif (1928-1951), un volontariat politique au service du Parti conservateur?
}

Volunteering for Political Ends? The Mobilisation of Conservative Women and

Non-Partisan Associations (1928-51)

\section{Clarisse Berthezène}

\section{CpenEdition}

Journals

Édition électronique

URL : http://journals.openedition.org/rfcb/1432

DOI : $10.4000 /$ rfcb. 1432

ISSN : 2429-4373

Éditeur

CRECIB - Centre de recherche et d'études en civilisation britannique

\section{Référence électronique}

Clarisse Berthezène, «L'Engagement des femmes dans le monde associatif (1928-1951), un volontariat politique au service du Parti conservateur? ", Revue Française de Civilisation Britannique [En ligne], XXII-3 | 2017, mis en ligne le 05 juillet 2017, consulté le 19 avril 2019. URL : http:// journals.openedition.org/rfcb/1432; DOI : 10.4000/rfcb.1432

Ce document a été généré automatiquement le 19 avril 2019

\section{C)}

Revue française de civilisation britannique est mis à disposition selon les termes de la licence Creative Commons Attribution - Pas d'Utilisation Commerciale - Pas de Modification 4.0 International. 


\title{
L'Engagement des femmes dans le monde associatif (1928-1951), un volontariat politique au service $\mathrm{du}$ Parti conservateur?
}

\author{
Volunteering for Political Ends? The Mobilisation of Conservative Women and \\ Non-Partisan Associations (1928-51)
}

Clarisse Berthezène

\section{Introduction}

1 Un grand nombre d'associations d'entraide créées et animées par des femmes virent le jour pendant la Première Guerre mondiale. Il s'agissait avant tout de participer à l'effort de guerre. L'action sociale, entendue dans son sens le plus large, se développa pendant les années 1920 et 1930 au travers d'une multiplicité d'associations très diverses dans leurs formes et dans leurs objets ${ }^{1}$. Cette nébuleuse associative, en principe libre de toute attache politique, entretenait pourtant des relations multiformes et souvent ambiguës avec des partis politiques, notamment le Parti conservateur, qui cherchaient à les instrumentaliser. Cet espace intermédiaire aux contours indéfinis ${ }^{2}$, aux lisières des partis et des appartenances religieuses était considéré comme une pièce essentielle dans les rouages de la vie politique britannique. Une citoyenneté active fondée sur le sens du service rendu à la communauté était au cœur même de la démocratie. Mais, simultanément, ce monde associatif était perçu comme un concurrent potentiel ou même une menace par les partis politiques. Les Representation of the People Acts de 1918 et 1928 qui élargirent le suffrage universel et donnèrent le droit de vote aux femmes eurent pour conséquence de multiplier le corps électoral par quatre ${ }^{3}$. Ce quadruplement du nombre des électeurs fut le point de départ d'une véritable course entre les partis pour former aussi bien que façonner ce nouvel électorat, le convaincre tout autant que le séduire, reflétant les tensions entre éducation et propagande au sein de chaque parti. 
L'historiographie a montré combien le Parti conservateur fut prompt à répondre aux défis de la démocratisation par des politiques ciblées vers les électeurs et électrices, en fonction de leur sexe et de leur appartenance sociale, régionale et religieuse ${ }^{4}$. L'engagement politique des femmes conservatrices au sein de nombreuses associations d'entraide semble avoir joué un rôle non négligeable dans le succès électoral des conservateurs pendant l'entre-deux-guerres. Le monde associatif était-il l'antichambre du monde politique? Après 1945, l'étude du Women's Voluntary Services, organisation regroupant un assez grand nombre de ces associations a mis en évidence l'importance de l'échelon local dans cette mobilisation. L'étude de l'articulation entre le secteur associatif et l'État notamment à l'occasion de la mise en place de l'État Providence donne un éclairage nouveau sur le rôle joué par les femmes dans l'architecture de cette nouvelle construction.

\section{La « nouvelle philanthropie »}

2 Les défenseurs de la «Big Society» de David Cameron voient dans le secteur associatif un élément clé dans le dispositif de lutte contre l'omnipotence de l'État. L'âge d'or du secteur associatif serait le $\mathrm{xIX}^{\mathrm{e}}$ siècle, la première partie $\mathrm{du} \mathrm{xx}^{\mathrm{e}}$ étant celle d'un déclin marqué consécutif à la montée en puissance de l'État Providence. Ce thème est fréquemment développé par les adversaires de l'État Providence mais a été également soutenu par certaines recherches universitaires ${ }^{5}$. Pourtant une historiographie renouvelée dans les dix dernières années a montré combien l'État et le secteur associatif étaient dépendants l'un de l'autre depuis la fin du $\mathrm{XIX}^{\mathrm{e}}$ siècle $^{6}$. En effet, les recherches les plus récentes complétant des travaux plus anciens confirment la permanence en Grande-Bretagne d'une " économie mixte de protection sociale » (« a mixed economy of welfare »), impliquant à la fois des intervenants publics et privés, et ce, bien avant que se soit popularisé le concept de « frontière mouvante » entre public et privé cher à William Beveridge ${ }^{7}$.

3 En 1934, Elizabeth Macadam évoquait la "nouvelle philanthropie », qu'elle définissait comme un "partenariat unique " entre l'État et le secteur associatif, "un système de service social à la fois bénévole et statutaire [...] qui existe nulle part ailleurs " ${ }^{8}$. Elle voyait dans l'entre-deux-guerres une période de développement de la protection sociale et de l'organisation des relations entre l'État et les mouvements associatifs. Un rapport de 1952 sur le secteur associatif et l'État montre que la Première Guerre mondiale marqua un « tournant » dans l'histoire du bénévolat et explique que c'est pendant les années trente que le secteur associatif cessa d'être simplement un service de charité ou de mécénat de la bourgeoisie pour devenir un service rendu par des citoyens de toutes classes sociales 9 . En réponse au chômage massif des années trente, le volontariat se développa dans toutes les couches de la société, se « démocratisa » en quelque sorte. Le London County Council est un bon exemple de cette " économie mixte de protection sociale ». Une de ses composantes, le

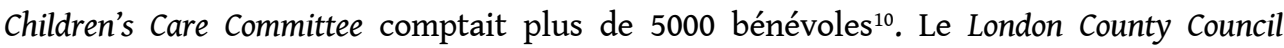
supervisait de nombreux hôpitaux organisés par des bénévoles. Une loi de 1936 imposa au London County Council le recours au service de sages-femmes salariées recrutées par les associations de volontaires ${ }^{11}$.

4 Ce volontariat était conçu comme « un travail qui forme le caractère et qui apporte une double récompense. Il enrichit à la fois ceux qui se mettent ainsi au service des autres et ceux qui reçoivent ${ }^{12}$.» L'idée de former le caractère («character-building») des citoyens et surtout des citoyennes en inculquant le sens du service n'était pas entièrement nouvelle. Elle faisait 
partie de la tradition philanthropique du XIX ${ }^{e}$ siècle, mais elle fut reprise de manière efficace par le Parti conservateur. Dans ses discours, Stanley Baldwin mettait l'accent sur les devoirs liés aux privilèges de la citoyenneté, et notamment celui de s'occuper de sa communauté. Baldwin expliquait ainsi: "L'affirmation des droits des peuples n'a encore jamais donné de pain à ces peuples. Seul l'accomplissement de leurs devoirs peut couronner de succès les expériences politiques que nous avons poussées plus loin que tous les autres peuples $d u$ monde ${ }^{13}$. » Parlant des " friendly societies », des sociétés de secours mutuel créées au XIX siècle pour jouer le rôle d'assurances contre le chômage et la maladie ${ }^{14}$, il déclarait :

Ce n'est pas parler en l'air que d'essayer de comprendre ce que représente le grand mouvement des sociétés de secours mutuel. [...] Il a été un instrument important durant une période de changements rapides en enseignant à nos concitoyens quelques-uns des grands principes qui doivent guider leurs vies [...]. Tout l'esprit du mouvement est dans le service des autres [...]. En enseignant à notre peuple l'esprit de service qu'inculquent de leur côté les sociétés de secours mutuel, nous jouons notre rôle et rendons notre démocratie plus généreuse et plus efficace ${ }^{15}$.

5 Les sociétés de secours mutuel ne dépendaient ni d'entreprises philanthropiques ni de l'État ; elles incarnaient l'idéal de la citoyenneté porté par les libéraux au XIX siècle et par les conservateurs au xxe siècle. Pour les libéraux, il s'agissait de l'autonomie de l'individu, organisant son propre système d'assurance, pour les conservateurs de Baldwin, l'accent était mis sur l'idéal communautaire des associations. Le secteur associatif était présenté et perçu comme le liant d'une société et le cœur de la démocratie. "'L'Association Libre' est un mode d'expression propre à la Démocratiie ${ }^{16}$." Libre de toute affiliation politique ou religieuse, conçu comme un lieu intermédiaire entre l'État et le citoyen, le secteur associatif était le terreau à partir duquel la citoyenneté, et notamment la citoyenneté nouvellement acquise par le droit de vote en 1918 et en 1928, pouvait s'exprimer. Arthur Bryant, historien populaire et chantre du Parti conservateur, s'adressant à la conférence annuelle du National Council for Social Service en 1946 mit sur le même plan le «selfgovernment » local et les organisations de volontaires et expliqua que la Grande-Bretagne avait gagné la guerre grâce à sa démocratie fondée sur des siècles de service volontaire (« voluntary service ») dans les paroisses. C'était également une manière de lutter contre le risque de contamination par les modèles continentaux de centralisation étatique, napoléonien ou bismarckien. La vie associative devait servir non seulement à la formation civique et démocratique mais être un tremplin pour encourager les citoyens à s'engager dans la vie de la cité. Considérée comme une arme essentielle dans la lutte contre le fascisme, il s'agissait de créer "une certaine forme de service public et d'aider ainsi à la création d'une démocratie active et non passive» ${ }^{17}$, engagée «dans différentes formes de service public, obligatoire ou volontaire $»^{18}$.

6 L'accent mis sur le volontariat dans la réflexion sur la citoyenneté après 1928 et pendant la seconde guerre mondiale a conduit à sous-estimer le rôle joué par les partis politiques au sein de ces organisations en principe non partisanes. En effet, à droite comme à gauche, le mouvement associatif conduit et animé par des volontaires était considéré comme un rouage essentiel de la démocratie et l'arme la plus efficace pour lutter contre les dictatures, la figure du volunteer incarnant le citoyen modèle par excellence. G.D.H. Cole, défenseur d'un socialisme libéral et participatif, voyait dans le développement d'un volontariat travaillant en lien étroit avec l'Etat la promesse d'une ère nouvelle plus égalitaire ${ }^{19}$. Pour autant, si cet espace intermédiaire semblait non seulement accepté, mais également protégé et même célébré par les partis politiques, ces derniers, notamment le Parti conservateur, étaient bien conscients du conflit d'influence qui était à 
l'œuvre au sein de ces associations. Pour les conservateurs, il était essentiel d'être présents dans chacune de ces associations et de savoir ce qui s'y disait. "Unpolitical politics", la politique non partisane, revêtait une importance nouvelle avec l'élargissement du suffrage. Baldwin expliquait: "Je suis heureux que le mouvement des sociétés de secours mutuel ignore la politique [...]. Je suis reconnaissant que ce mouvement ne se soit jamais laissé prendre en otage par les machines des partis politiques ${ }^{20}$. " C'est pourtant le même Baldwin qui souligna l'importance du secteur associatif dans sa quête non désintéressée $\mathrm{du}$ vote ouvrier féminin. C'est dans ce contexte que les militantes conservatrices furent amenées à jouer un rôle particulier.

\section{Féminisme et citoyenneté}

7 Pour de nombreuses femmes, le langage de la citoyenneté et l'engagement dans une association étaient également une manière de promouvoir la cause des femmes sans adopter pour autant une posture ouvertement féministe. Descendant en droite ligne des sociétés de philanthropie du XIX siècle, dans lesquelles il était admis que les femmes jouent un rôle important, le volontariat féminin de l'entre-deux-guerres permettait une mobilisation efficace sur de nombreuses questions politiques et sociales. Le Parti conservateur se présentait depuis le tournant du siècle comme le parti « du foyer et de la famille» («Home and Hearth») dans son combat contre le socialisme. Dans ce contexte, le féminisme était perçu comme synonyme de destruction du foyer et perversion des mœurs. A l'exception de certaines conservatrices qui se revendiquaient ouvertement féministes, Lady Astor en étant un exemple célèbre, la plupart d'entre elles adoptaient le langage de la citoyenneté et des droits et des devoirs, qui leur permettait de faire valoir ce qu'elles faisaient pour les autres, en étant, selon les termes du Premier Ministre

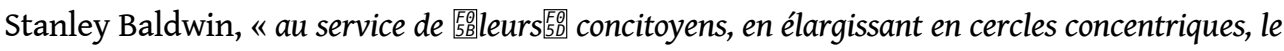

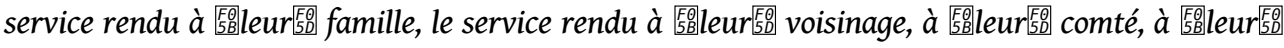

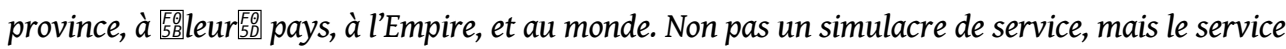

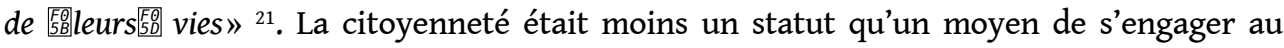
service de la nation ${ }^{22}$. Elle ne relevait pas d'un droit universel mais du caractère particulier d'une nation et de l'engagement de l'individu au sein de sa communauté. Pour les femmes, elle était comprise comme l'extension de leurs devoirs domestiques à leur quartier, leur ville, leur pays, en cercles concentriques, selon l'expression de Baldwin.

8 Les organisations de volontaires qui fleurirent pendant la Première Guerre mondiale eurent pour ambition, une fois la guerre finie, de poursuivre leur action à l'abri de la politique et des partis ${ }^{23}$. S'engageant dans l'organisation d'associations de bénévoles de toutes sortes (des service clubs, aux associations de formation à la citoyenneté, telle l'Association for Education in Citizenship, aux associations pacifistes comme la League of Nations Union, ou aux associations rurales comme les Women's Institutes), les femmes investirent nombreuses ces nouvelles institutions qui participaient à la vie publique. Les associations exclusivement féminines leur donnaient l'opportunité de s'exprimer librement, sans risque de voir la légitimité intellectuelle de leurs propos remise en cause par une présence masculine ${ }^{24}$.

9 L'émergence de lieux au sein desquels il était possible de s'exprimer en dehors des partis politiques, compris comme des espaces de pouvoir alternatifs, fut d'abord considérée comme une menace pour les partis ${ }^{25}$. Cependant, la présence importante de militantes conservatrices qui avaient investi ces associations pendant la guerre, donnant de leur 
temps et de leur énergie pour soutenir l'effort de guerre convainquit le Parti conservateur qu'il était possible d'utiliser ces associations pourtant non partisanes pour attirer un électorat plus large. L'accueil des réfugiés belges pendant la Première Guerre mondiale, par exemple, fut initialement organisé par l'association conservatrice Women's Unionist Tariff Reform Association (WUTRA) ${ }^{26}$. En 1917, les Women's Institutes et les organisations intéressées par le travail des femmes dans les campagnes étaient des associations phares de l'effort de guerre. Elles étaient placées sous la tutelle de la Women's Branch of the Board of Agriculture dont la conservatrice Edith Lyttelton était directrice adjointe.

10 Pour Mary Maxse qui dirigeait la WUTRA, ces associations étaient un moyen efficace de combattre ce qu'elle percevait comme l'idéologie de lutte des classes promue par les Socialistes. Les associations de l'époque édouardienne s'intéressaient surtout à la formation des femmes qui souhaitaient participer aux élections en faisant du porte-àporte. A partir des années 1920, ces associations prirent la forme de réunions plus informelles afin d'attirer les nouvelles électrices censées avoir peu d'intérêt pour la politique. En 1919, cherchant à imiter la culture politique des Women's Institutes, Annie Chamberlain, la femme du futur Premier ministre Neville Chamberlain, créa des Unionist Women's Institutes dans les circonscriptions défavorisées de Birmingham. Ces « instituts " offraient un lieu de rencontre pour les ouvrières, très différent des salons des demeures aristocratiques, caractéristiques de la culture sociale des conservatrices avant la Première Guerre mondiale ${ }^{27}$. Il s'agissait à la fois de centres sociaux et de lieux où l'on pouvait débattre de la citoyenneté. Des discussions politiques sur des sujets simples telles que « l'ABC de l'Unionisme » ou «Ce que fait la municipalité pour les enfants » étaient proposées ${ }^{28}$. Ce modèle de militantisme contribua à ce que les associations féminines soient au cœur de l'organisation locale et populaire du Parti, notamment dans les quartiers pauvres où les travaillistes étaient très présents. Les instituts d'Annie Chamberlain sont l'exemple même de la manière dont les conservatrices s'inspirèrent des associations nées de la guerre pour proposer des lieux de sociabilité ouverts à tous, ou plutôt à toutes, et un engagement en apparence apolitique.

11 C'est ainsi qu'en 1928, la Women's Unionist Organisation expliquait qu'il était essentiel que le Parti conservateur « adopte tous les moyens possibles pour intéresser les femmes du pays aux affaires publiques $»^{29}$. A titre d'exemple, elle encourageait la création de Clubs Fuchsia : "Les Clubs Fuchsia ne sont pas des clubs au sens ordinaire du mot, mais des lieux de réunion ouverts à toutes. Le sens du terme "Fuchsia" vient des mots "Friendship", "Understanding", "Cheerfulness", "Helpfulness", "Sympathy", "Instruction" et "Amusement". Les objectifs de ce club sont doubles : être un lieu d'éducation et de sociabilité. Le but est de diffuser la propagande 周:conservatrice咸 aux électrices de tous les partis ${ }^{30}$. 》

12 Cependant, il ne s'agissait pas simplement de proposer de nouveaux lieux de sociabilité, mais également «d'infiltrer", selon leur propre terme, les associations existantes. Les réseaux et les liens tissés par les conservatrices au sein de fondations philanthropiques et de charités avaient toujours beaucoup contribué à l'intégration du Parti conservateur au sein des communautés à l'échelon local ${ }^{31}$. Le 30 septembre 1936, lors d'une réunion de la National Society of Women Organisers du Parti conservateur, il fut clairement énoncé l'importance d'être présente aux réunions des différentes associations afin de suivre les débats et, éventuellement, de faire valoir le point de vue conservateur. Les activités de plusieurs associations étaient suivies de près, en particulier la Co-Operative Society, la League of Nations Union et le Peace Council ${ }^{32}$. De même, le Central Women's Advisory Committee 
(CWAC) du Parti conservateur avait des observateurs au sein de Over-thirty Association, Women's Freedom League, National Council for Equal Citizenship, London and National Society for Women's Service, Wayfarers' Sunday Association, Open Door Council, Six Point Group, National Council of Women and Women's Guild of Empire ${ }^{33}$. En mars 1937, lors d'une réunion du CWAC du Parti conservateur, il fut de nouveau rappelé l'urgence de s'investir davantage dans les échelons locaux des associations ${ }^{34}$. Il fut également demandé à celles qui étaient membres de syndicats ou de sociétés co-opératives d'être plus actives afin de «libérer ces sociétés des politiques partisanes $»^{35}$. La bataille pour s'attirer les faveurs des nouveaux électeurs et électrices faisait rage et le monde associatif en était un enjeu important.

\section{Les Women's Voluntary Services}

13 C'est dans ce contexte que s'inscrit l'histoire des Women's Voluntary Services (WVS), créés en 1938 par Lady Reading à la demande du Ministère de l'Intérieur, pour préparer la population civile à la menace d'une guerre, notamment à l'éventualité de bombardements et d'évacuation de la population des villes. Il s'agissait de réunir les associations existantes et de coordonner leur travail. Les WVS réunirent près d'un million de volontaires pendant la guerre. Ils étaient découpés en onze régions de défense civile comprenant un organisateur volontaire et un administrateur rémunéré pour contrôler l'ensemble des volontaires de chaque région. Ils étaient financés par l'Etat mais s'appuyaient sur le travail de volontaires, la hiérarchie au niveau local étant largement calquée sur la hiérarchie déjà existante au sein de leurs organisations membres, en particulier les Women's Institutes, les Townswomen's Guilds et le guidisme. Ils étaient organisés de manière pyramidale, et envoyaient des instructions depuis les bureaux de Londres. Après la guerre, ils continuèrent de mobiliser des volontaires dans les hôpitaux et pour l'aide aux personnes âgées, mais également en cas d'urgences nationales, comme pendant les inondations de 1946, 1952 et 1953. Leurs principaux terrains d'action étaient alors les services d'aide à domicile en partenariat avec les municipalités (Home-help service et meals-on-wheels), le relogement des réfugiés polonais, les questions d'alimentation et de rationnement. Dans Problems of Social Policy, Richard Titmuss faisait l'éloge des WVS et d'autres organisations de volontaires qui avaient contribué à transformer les centres d'accueil « lugubres et hostiles » en septembre 1939 en « des gîtes d'hébergement sympathiques et accueillants $»^{36}$. L'efficacité des WVS, leur approche humaine et leur esprit innovant étaient mis en valeur et Lady Reading obtint du gouvernement Attlee que leur financement soit poursuivi en temps de paix.

14 Si les WVS avaient les faveurs des municipalités et du gouvernement, ils s'étaient fait des ennemis parmi les autres associations de volontaires, qui avaient accepté de les soutenir pendant la guerre uniquement parce qu'elles avaient reçu l'assurance qu'ils fermeraient une fois la guerre finie. Une des raisons principales expliquant l'hostilité envers les WVS tenait au fait que leurs frais de gestion et de fonctionnement étaient couverts par le gouvernement. Après la guerre, ils continuèrent de percevoir des financements de l'État. Il ne s'agissait pas d'une aide ponctuelle pour un projet spécifique, ce dont toute association pouvait bénéficier, mais d'un financement régulier. Aucune autre association ne recevait ce type d'aide. Les WVS étaient une organisation « anormale, soutenue par l'État et pourtant bénévole». C'est précisément ce que revendiquait leur fondatrice, Lady Reading, qui les décrivait non pas comme « une organisation de volontaires financée par l'État, 
mais comme un service d'État fourni par des volontaires $»^{37}$. Elle aimait à penser que les WVS jouaient le rôle d'une interface entre le secteur associatif dans son ensemble et l'État.

Lady Reading n'était pas membre du Parti conservateur, les WVS étant officiellement apolitiques. Il n'en reste pas moins que de nombreuses dirigeantes des WVS étaient d'éminentes personnalités du Parti. Le recoupement entre leurs membres ne pouvait pas passer inaperçu ${ }^{38}$. Marjorie Maxse, directrice générale de l'organisation du Parti conservateur (chief organisation officer), était l'une des trois vice-présidentes des WVS. Les critiques adressées aux WVS portaient à la fois sur le financement par l'État mais également sur la structure hiérarchique jugée peu démocratique de l'association. En avril 1948, le Women's Group on Public Welfare (WGPW) ${ }^{39}$ devint le fer de lance d'une opposition féroce aux WVS et publia un mémorandum dans lequel il était déclaré que " (a) la différence entre une organisation de volontaires authentique et une organisation qui agit au nom du gouvernement doit être clairement définie. (b) Si les WVS doivent être considérées comme une organisation de volontaires, il leur faut une constitution de nature démocratique comme celle des autres organisations ${ }^{40}$. » Le manque de fonctionnement démocratique des WVS et la nature autocrate de sa présidente furent fustigés par les autres associations. En réponse à ces accusations, Lady Reading insistait sur la modernité des WVS et sur le côté désuet des associations de volontaires d'avant-guerre. Elle expliquait en effet que les WVS avaient réussi la transition d'un modèle associatif des années trente, qu'elle qualifiait de «service de charité bénévole aléatoire à l'ancienne", vers un modèle "moderne" dans lequel ils agissaient comme " une courroie de transmission entre les objectifs énoncés au niveau central et le plus petit échelon afin que l'idée centrale se traduise en action au niveau local »" L'époque du mécénat et de la philanthropie était révolue et l'État devait désormais couvrir les frais de fonctionnement de cette armée de volontaires. Les Women's Institutes et le WGPW craignaient que l'indépendance du secteur associatif soit incompatible avec le financement par l'État. Un long débat s'engagea entre 1947 et 1951 sur l'avenir du secteur associatif et sa place au sein de l'État-Providence, et plus précisément sur le rôle des WVS dans ce nouvel ensemble.

Le 22 juin 1949 eut lieu à la Chambre de Lords, sur la proposition de Lord Samuel, un débat sur la «Voluntary Action for Social Progress ». Ce débat fut l'occasion de réaffirmer le consensus selon lequel «l'esprit du volontariat est l'âme même de la démocratie "42, que le secteur associatif est "un pont entre les différentes classes sociales de la communauté »" L'esprit du volontariat fut décrit comme étant par essence "créatif», permettant le dépassement de l'antagonisme entre action publique et poursuite des intérêts égoïstes de l'individu. Lord Pakenham défendait l'idée d'un volontariat rendu plus efficace par la proximité de Whitehall et d'autorités publiques locales, et d'un État bénéficiant de l'énergie et du pouvoir créatif dont seuls les individus ensemble pouvaient faire preuve ${ }^{44}$.

Suivant en cela les recommandations de Lord Beveridge dans son rapport de 1949 The Evidence for Voluntary Action ${ }^{45}$, Lady Reading militait pour une forme renouvelée de volontariat, rassurant par là-même, ceux parmi les conservateurs qui prédisaient le triomphe de l'« étatolatrie » et, du même coup, la fin du secteur associatif, et in fine de la démocratie. En 1951, il fut voté que les WVS seraient financés par l'État de manière permanente. Ceux-ci étaient parvenus à faire accepter un modèle nouveau de volontariat, montrant ainsi aux conservateurs, en pleine déroute après la défaite électorale de 1945, que les femmes de leur parti étaient à la pointe d'une certaine forme de "modernité »46. Elles avaient également contribué à maintenir inchangée une structure de classe au sein 
de l'association, organisée de manière strictement hiérarchique, les femmes des classes aisées étant à la tête de la pyramide sociale ${ }^{47}$.

\section{Conclusion}

La mise en place de ce qui a été appelé l'État-Providence ne marqua en rien la fin du mouvement bénévole ni du "partenariat unique » entre l'État et le secteur associatif, qu'Elizabeth Macadam appelait de ses voeux. Les années 1940 et 1950 se caractérisèrent, au contraire, par des débats animés au sujet de la nature du volontariat et du rôle respectif des associations et de l'État. Les chiffres montrent que le volontariat ne cessa de croître dans les années d'après-guerre ${ }^{48}$. Nombre d'organisations féminines s'engagèrent dans ce débat, aux côtés du National Council of Social Service (NCSS) et des autorités locales. Entre 1945 et 1947, la question porta principalement sur le passage d'un volontariat de temps de guerre à un modèle associatif en temps de paix. Le rapport de 1948 intitulé Voluntary Action, dans lequel Lord Beveridge évoquait la célèbre "frontière mouvante " entre les secteurs privé et public, portait également sur la réforme du statut des volontaires et sur leur place au sein des services d'aide sociale organisés par l'État ${ }^{49}$. Un article daté de mars-mai 1948 dans la Social Service Review, revue du National Council of Social Service (NCSS) expliquait qu'il s'agissait avant tout de savoir comment répartir le travail entre un organisme public, central ou local,

[organisme] dont l'argent provient des impôts des citoyens, et qui a de ce fait, des comptes à rendre sur ses dépenses, de sorte que même si cet organisme public est progressiste, il risque inévitablement de se limiter à une administration sans risque, obsédée par «l'uniformité de traitement » jusqu'à en devenir stéréotypée. Le secteur associatif possède une liberté d'action bien plus grande ainsi que la possibilité d'expérimenter des approches beaucoup plus variées et intimes de l'humain. [...] Utilisée intelligemment, l'action bénévole peut être le fer de lance du progrès social, soutenue par l'État et son financement ${ }^{50}$.

C'est dans ce contexte que les Women's Voluntary Services occupèrent une place singulière dans le paysage associatif de l'immédiat après-guerre, incarnant une «modernité conservatrice ", selon l'expression d'Alison Light. Les femmes des WVS trouvèrent un mode de fonctionnement en lien avec l'État plus adapté aux besoins des années 1940, montrant tout à la fois leur inventivité et leur capacité d'adaptation. Si la philanthropie pratiquée par les femmes au XIX ${ }^{e}$ siècle a souvent été décrite comme une opportunité de prise de pouvoir par les femmes des classes moyennes, dans les années 1940, le monde associatif s'ouvrait aux femmes de tous milieux sociaux, mais les WVS maintenaient à leur tête un groupe de femmes issu des classes aisées et imposant une organisation pyramidale. Le succès du Parti conservateur dans l'entre-deux-guerres tient pour beaucoup à sa capacité de construire et de développer des organisations féminines partisanes et non partisanes, en créant des réseaux de sociabilité au niveau local, permettant aux conservateurs de s'intégrer dans différentes communautés. Les WVS offrent un exemple de la manière dont certaines associations rebondirent après la défaite électorale des conservateurs en 1945 et s'adaptèrent aux défis de l'après-guerre, préparant leurs succès électoraux des années 1950.

Clarisse Berthezène est maîtresse de conférences en civilisation britannique à l'Université Paris Diderot (LARCA UMR 8225). Elle a consacré de nombreux articles et ouvrages à l'histoire du Parti conservateur britannique, dont Training minds for the war of ideas. The Conservative Party, Ashridge College and the cultural politics of 
Britain, 1929-54 (Manchester University Press, 2015) et, en co-direction avec JeanChristian Vinel, Conservatismes en mouvement. Une approche transnationale (Editions de l'EHESS, octobre 2016).

\section{BIBLIOGRAPHIE}

BALDWIN Stanley, « Democracy and the Spirit of Service », Londres, 4 décember 1924, in On England and Other Addresses, Londres, Philip Allan \& Co., 1926.

BALDWIN Stanley, Service of Our Lives: Last Speeches as Prime Minister, London, Hodder \& Stoughton Ltd, 1937,

BALL Stuart, Portrait of a Party: The Conservative Party in Britain 1918-1945, Oxford University Press, 2013

BEAUMONT Caitriona, Housewives and Citizens. Domesticity and the Women's Movement in England, 1928-64, Manchester, Manchester University Press, 2013

BERTHEZÈNE Clarisse, Training Minds for the War of Ideas. Ashridge College, the Conservative Party and the Cultural Politics of Britain, 1929-1954, Manchester, Manchester University Press, 2015.

BEVERIDGE William, Voluntary Action : A Report on Methods of Social Advancement, Londres, Allen and Unwin Ltd, 1948.

BEVERIDGE William et WeLls A.J., The Evidence for Voluntary Action, Londres, Allen and Unwin Ltd, 1949.

BINGHAM Adrian, Gender, Modernity, and the Popular Press in Inter-War Britain, Oxford, Oxford University Press, 2004.

BREWIS Georgina, « Towards a New Understanding of Volunteering in England Before 1960 ? ", Institute for Volunteering Research « Back to Basics », Series : Paper Two, 2012.

COLE G.D.H., " Mutual Aid Movements in their relation to Voluntary Social Service », in BOURDILlon Anne F. C. (dir.), Voluntary Social Services. Their Place in the Modern State, Londres, Methuen \& Co., 1945.

FINLAYSON Geoffrey, Citizen, State and Social Welfare in Britain, 1830-1990, Oxford, Clarendon Press, 1994.

HARRIS Jose (dir.), Civil Society in British History : Ideas, Identities, Institutions, Oxford, Oxford University Press, 2003.

HARRIS Ralph et SELDON Arthur, Welfare without the State : A Quarter of a Century of Suppressed Public Choice, Londres, Institute of Economic Affairs, 1987.

HILTON Matthew et McKay James (dir.), The Ages of Voluntarism. How We Got to the Big Society, Oxford, Oxford University Press, 2011.

HINTON James, Women, Social Leadership and the Second World : Continuities of Class, Oxford, Oxford University Press, 2002 
HOPKINS Eric, Working-Class Self-Help, Londres, U.C.L. Press, 1995.

JARVIS David, « Mrs Maggs and Betty. The Conservative Appeal to Women Voters in the 1920s », Twentieth Century British History, vol. 5, n² 2, 1994, p. 129-152.

JARVIS David, «British Conservatism and Class Politics in the 1920s », English Historical Review, vol. 110, 1996, p. 59-84.

JARVIS David, « "Behind Every Great Party”: Women and Conservatism in Twentieth Century Britain », in VICKERY Amanda, Women, Privilege, and Power: British Politics, 1750 to the Present, Stanford, Stanford University Press, 2002, p. 305.

LIGHT Alison, Forever England : Femininity, Literature and Conservatism Between the Wars, Londres, Routledge, 1991.

MACADAM Elizabeth, The New Philanthropy: A Study of the Relations between the Statutory and Voluntary Social Services, Londres, George Allen and Unwin, 1934.

MCCARTHY Helen, « Parties, Voluntary Associations and Democratic Politics in Interwar Britain », The Historical Journal, 50/4, 2007, p. 891-912.

MCCARTHY Helen, « Service Clubs, Citizenship and Equality : Gender Relations and Middle-Class Associations in Britain Between the Wars », Historical Research, vol. 81, n²13, août 2008.

OPPENHEIMER Melanie et DEAKIN Nicholas (dir.), Beveridge and Voluntary Action in Britain and the Wider British World, Manchester, Manchester University Press, 2011.

PROCHASKA Franck, Christianity and Social Service in Modern Britain : The Disinherited Spirit, Oxford, Oxford University Press, 2006.

ROCHESTER Colin et al., (dir.), Understanding the Roots of Voluntary Action. Historical Perspectives on Current Social Policy, Sussex, Sussex Academic Press, 2011.

THACKERAY David, Conservatism for the Democratic Age. Conservative Cultures and the Challenge of Mass Politics in Early Twentieth-Century England, Manchester, Manchester University Press, 2013.

THACKERAY David, «Faire de la politique à l'heure de la démocratie. Libéralisme et conservatisme populaire en Grande-Bretagne, 1906-1924 », Vingtième Siècle, n¹20, 2013, p. 57-69.

THACKERAY David, « Home and Politics : Women and Conservative Activism in Early TwentiethCentury Britain », Journal of British Studies, vol. 49, n4, 2010, p. 826-48.

THANE Pat, « The Ben Pimlott Memorial Lecture 2011. The "Big Society" and the "Big State" : Creative Tension or Crowding Out?», Twentieth Century British History, vol. 23, n³, 2012, p. 408-429.

TITMUSs Richard, Problems of Social Policy, Londres, HMSO, 1950.

WEIGHT Richard et BEACH Abigail (dir.), The Right to Belong : Citizenship and Identity in Britain 1930-1960, Londres, I. B. Tauris, 1998.

\section{NOTES}

1. WEIGHT Richard et BEACH Abigail (dir.), The Right to Belong: Citizenship and Identity in Britain 1930-1960, Londres, I. B. Tauris, 1998 ; HARRIS Jose (dir.), Civil Society in British History: Ideas, Identities, Institutions, Oxford, Oxford University Press, 2003; MccARTHY Helen, «Parties, Voluntary Associations and Democratic Politics in Interwar Britain ", The Historical Journal, $\mathrm{n}^{\circ}$ 
50/4, 2007, p. 891-912; HILTON Matthew et McKay James (dir.), The Ages of Voluntarism. How We Got to the Big Society, Oxford, Oxford University Press, 2011.

2. Sur les questions de terminologie et de définitions, voir l'introduction de HARRIS Jose (dir.), «Introduction; Civil Society in British History : Paradigm or Peculiarity? ", Civil Society in British History, op. cit., p. 1-12 et celle de HILTON Matthew et McKAY James (dir.), The Ages of Voluntarism, op.cit, p. 1-26.

3. Le "Fourth Reform Act » de 1918 accorde le droit de vote et l'éligibilité au Parlement aux hommes de plus de 21 ans et aux femmes de plus de 30 ans qui paient des impôts locaux ou dont l'époux en paie. Le "Fifth Reform Act» de 1928 accorde le même droit de vote aux femmes qu'aux hommes : les femmes de plus de 21 ans peuvent désormais voter.

4. JARvis David, "Mrs Maggs and Betty. "The Conservative Appeal to Women Voters in the 1920s ", Twentieth Century British History, vol. 5, n. 2, 1994, p. 129-152 ; JARVIS David, «British Conservatism and Class Politics in the 1920s ", English Historical Review, vol. 110, 1996, p. 59-84; BINGHAM Adrian, Gender, Modernity, and the Popular Press in Inter-War Britain, Oxford, Oxford University Press, 2004 ; BALL Stuart, Portrait of a Party: The Conservative Party in Britain 1918-1945, Oxford University Press, 2013 ; THACKERAY David, Conservatism for the Democratic Age. Conservative cultures and the Challenge of Mass Politics in Early Twentieth-Century England, Manchester, Manchester University Press, 2013; BERTHEŻ̀nE Clarisse, Training Minds for the War of Ideas. Ashridge College, the Conservative Party and the Cultural Politics of Britain, 1929-1954, Manchester, Manchester University Press, 2015.

5. Voir par exemple HARRIS Ralph et SELDON Arthur, Welfare without the State: A Quarter of a Century of Suppressed Public Choice, Londres, Institute of Economic Affairs, 1987; PROCHASKA Franck, Christianity and Social Service in Modern Britain: The Disinherited Spirit, Oxford, Oxford University Press, 2006.

6. HILTON Matthew et McKAy James (dir.), The Ages of Voluntarism, op. cit.; THANE Pat, "The Ben Pimlott Memorial Lecture 2011. The "Big Society" and the "Big State": Creative Tension or Crowding Out?", Twentieth Century British History, vol. 23, n 3, 2012, p. 408-429 ; BREWIS Georgina, "Towards a New Understanding of Volunteering in England Before 1960 ?», Institute for Volunteering Research « Back to Basics », Series : Paper Two, 2012.

7. FINLAYSON Geoffrey, Citizen, State and Social Welfare in Britain, 1830-1990, Oxford, Clarendon Press, 1994 ; HARRIS Jose (dir.) Civil Society in British History. Ideas, Identities, Institutions, Oxford, Oxford University Press, 2003 ; ROCHESTER Colin et al., (dir.), Understanding the Roots of Voluntary Action. Historical Perspectives on Current Social Policy, Sussex, Sussex Academic Press, 2011 ; opPenHeIMER Melanie et DEAKIN Nicholas (dir.), Beveridge and Voluntary Action in Britain and the Wider British World, Manchester, Manchester University Press, 2011.

8. MACADAm Elizabeth, The New Philanthropy: A Study of the Relations between the Statutory and Voluntary Social Services, Londres, George Allen and Unwin, 1934, p. 285 ; BEVERIDGE William , Voluntary Action : A Report on Methods of Social Advance, Londres, George Allen and Unwin, 1948.

9. "Voluntary Service and the State: A Study of the Needs of the Hospital Service " (Londres, NCSS and King Edward's Hospital Fund for London, 192), p. 14, cité dans BREWIS Georgina, " Towards a new understanding of volunteering in England before 1960 ? », op. cit., p. 8.

10. Discours présidentiel de Lord SNELL, président sortant, Social Service Review, XX, n 8, août 1939, p. 257.

11. Social Service Review, Vol XIX, $n^{\circ} 2$, février 1938.

12. Discours présidentiel de Lord SNELL, président sortant, Social Service Review, XX, $\mathrm{n}^{\circ} 8$, août 1939, p. 257.

13. BALDWIN Stanley, "Democracy and the Spirit of Service », Londres, 4 décembre 1924, in On England and Other Addresses, Londres, Philip Allan \& Co., 1926, p. 71. 
14. L'individu payait une cotisation hebdomadaire afin de bénéficier de ces assurances. Les «friendly societies » assuraient, par exemple, un enterrement au cimetière et non dans la fosse commune. Voir HOPKINS Eric, Working-Class Self-Help, Londres, U.C.L. Press, 1995.

15. BALDWIN Stanley à Stourport, 18 février 1924, in BALDWIN Stanley, On England and Other Addresses, p. 263-264.

16. "The Voluntary Movement in Great Britain in 1937 ». Paper prepared by the Vice-Chairman for consideration by the Executive Committee at its meeting on July $22^{\text {nd }} 1937$, LMA/4016/IS/ $\mathrm{A} / 01 / 107$.

17. Education Council Minutes, 5 octobre 1948, Minutes of the Education Council 1947-1949, Ashridge Papers.

18. Education Council : Sub-Committee on the Ashridge Circles and Publications, 5 octobre 1948, ibid.

19. COLE G.D.H., "Mutual Aid Movements in their relation to Voluntary Social Service ", in BoURdillon Anne F. C. (dir.), Voluntary Social Services. Their Place in the Modern State, Londres, Methuen \& Co., 1945.

20. BALDWIN Stanley à Stourport, 18 février 1924, in S. Baldwin, On England and Other Addresses, p. 263.

21. BALDWIN Stanley, Service of Our Lives: Last Speeches as Prime Minister, London, Hodder \& Stoughton Ltd, 1937, p. 144.

22. Beaumont Caitriona, Housewives and Citizens. Domesticity and the Women's Movement in England, 1928-64, Manchester, Manchester University Press, 2013, p. 42.

23. mcCARTHY Helen, "Parties, Voluntary Associations and Democratic Politics in Interwar Britain », p. 891-912.

24. MCCARTHY Helen, «Service Clubs, Citizenship and Equality: Gender Relations and MiddleClass Associations in Britain Between the Wars ", Historical Research, vol. 81, n²13, août 2008.

25. THACKERAY David, Conservatism for the Democratic Age ; THACKERAY David, «Faire de la politique à l'heure de la démocratie. Libéralisme et conservatisme populaire en Grande-Bretagne, 1906-1924 ", Dossier sous la direction de BERTHEZÈnE Clarisse et PURSEIGLE Pierre, «Déclin et résilience du libéralisme britannique ", Vingtième Siècle, ${ }^{\circ} 120,2013$, p. 57-69.

26. THACKERAY David, Conservatism for the Democratic Age.

27. THACKERAY David, op. cit.

28. THACKERAY David, "Home and Politics: Women and Conservative Activism in Early Twentieth-Century Britain ", Journal of British Studies, vol. 49, n²4, 2010, p. 842-845.

29. Handbook for Women Organisers and Workers, 1928, p. 1. CPA PUB 190/5.

30. Ibid, p. 115-116.

31. JARVIS David, «"Behind Every Great Party": Women and Conservatism in Twentieth Century Britain ", in VICKERY Amanda, Women, Privilege, and Power: British Politics, 1750 to the Present, Stanford, Stanford University Press, 2002, p. 305.

32. National Society of Women Organisers Minutes, 30 septembre, 1936, CCO 170/2/1/1.

33. Ibid, 10 février 1937, CCO 170/1/1/1.

34. Central Women's Advisory Committee Minute Book, 26 mars 1937, CCO 170/1/1/1.

35. Ibid.

36. TITMUSS Richard, Problems of Social Policy, Londres, HMSO, 1950, p. 267-268.

37. HINTON James, Women, Social Leadership and the Second World: Continuities of Class, Oxford, Oxford University Press, 2002, p. 217

38. On retrouve par exemple dans les deux organisations Lady Lloyd (Brecon), Evelyn Emmet, Mrs Weston (Chelmsford) Huxley et Lady Davidson. 
39. Créé en septembre 1939 sous l'égide de Margaret Bondfield (Travailliste) and Priscilla Norman (Conservatrice), le «Women's Group on Problems arising from Evacuation » change de nom et devient le «Women's Group on Public Welfare » en 1940.

40. Réunion du Women's Group on Public Welfare, 11 mai 1948, WGPW/27/4/48/70, CPA.

41. HINTON James, Women, Social Leadership and the Second World, p. 216.

42. Lord PAKENHAM, 22 juin 1949, Social Service, XXIII. N² , Sept-Nov 1949, p. 55-59.

43. L’Évêque de Sheffield, ibid.

44. Lord PAKenham, ibid, p. 59.

45. BeVERIDGe William et Wells A.J., The Evidence for Voluntary Action, Londres, Allen and Unwin Ltd, 1949.

46. Sur la «modernité conservatrice », voir LIGHT Alison, Forever England : Femininity, Literature and Conservatism Between the Wars, Londres, Routledge, 1991.

47. Le maintien des hiérarchies de classes au sein des WVS est le sujet principal du livre de HINTON James, Women, Social Leadership and the Second World, op. cit.

48. BREWIS Georgina, «Towards a new understanding of volunteering in England before 1960 ?», op. cit., p. 25.

49. BEVERIDGE William, Voluntary Action : A Report on Methods of Social Advancement, op.cit.

50. Social Service, mars-mai 1948, volume XXI, n4, p. 174.

\section{RÉSUMÉS}

Les femmes conservatrices jouèrent un rôle prépondérant au sein d'un monde associatif en pleine expansion après la Première Guerre mondiale. L'action sociale, entendue dans son sens le plus large, se développa pendant les années 1920 et 1930 au travers d'une multiplicité d'associations très diverses dans leurs formes et dans leurs objets. L'ampleur de cette nébuleuse associative pose la question de son instrumentalisation par les partis politiques, notamment le Parti conservateur. Le monde associatif fut en effet conçu comme un relais important (un concurrent aussi) des partis en quête d'électeurs. Occupant une place originale aux côtés de l'État dans la protection sociale, le monde associatif fut à ce titre en dialogue et en négociations permanentes avec lui. Les femmes conservatrices occupèrent ainsi une fonction politique importante à la fois comme courroies de transmission des partis, notamment au niveau local, et dans la conception, la négociation et l'organisation du système social en lien avec le pouvoir central.

The First World War saw the emergence of a variety of non-party organisations to aid the war effort, in which women played a crucial role. Social action, in its broadest sense, developed in the 1920 s and 1930s through the advent of a multiplicity of associations that were diverse in their forms and objectives. The establishment of new kinds of voluntary organisations raises questions as to their instrumentalisation by political parties, in particular the Conservative party. The political mobilisation of Conservative women within some of these associations seems to have played an important role in the Conservative electoral success of the interwar period. This paper will explore the role of Conservative women in the reworking of the relationship between the voluntary sector and the state after 1945. 
INDEX

Keywords : voluntary sector, Conservative Party, citizenship, women's organisations, Conservatism, WVS.

Mots-clés : secteur associatif, volontariat, Parti conservateur, citoyenneté, associations féminines, conservatisme, WVS

\section{AUTEUR}

\section{CLARISSE BERTHEZÈNE}

Université Paris Diderot (LARCA UMR 8225) 